Simulations and Measurements for a concept of powering CALICE-AHCAL at a train-cycled accelerator

This content has been downloaded from IOPscience. Please scroll down to see the full text. 2013 JINST 8 C01054

(http://iopscience.iop.org/1748-0221/8/01/C01054)

View the table of contents for this issue, or go to the journal homepage for more

Download details:

IP Address: 134.94.122.242

This content was downloaded on 07/08/2014 at 09:08

Please note that terms and conditions apply. 


\section{Simulations and Measurements for a concept of powering CALICE-AHCAL at a train-cycled accelerator}

\section{For the CALICE collaboration ${ }^{1}$}

\section{P. Göttlicher ${ }^{2}$}

DESY, Deutsches Elektronen-Synchrotron,

D-22607 Hamburg, Notkestrasse 85, Germany

E-mail: Peter.Goettlicher@desy.de

ABSTRACT: Improving calorimetry by usage of the particle-flow algorithm requires to record the details of the shower development. Therefore a high granularity analogue readout hadron calorimeter (AHCAL) with small sensors and with electronics handling the enormous amount of channels, $\approx 40000 / \mathrm{m}^{3}$, is required. Homogeneity is maintained by avoiding cooling tubes in the active volume and only cooling at the service end.

For this concept low power consumption per channel, $40 \mu \mathrm{W}$, is essential. Future linear $\mathrm{e}+\mathrm{e}^{-}-$collider designs, ILC or CLIC, foresee duty cycles for the bunch delivery. At ILC bunch trains of $1 \mathrm{~ms}$ duration are followed by long breaks of $200 \mathrm{~ms}$. Power cycling the front end electronics with the train structure can reduce power consumption by a factor 100 . However for a full scale CALICE-AHCAL switched currents reach magnitudes of kilo-amperes.

This paper describes the design chain from front end PCB's through to external power supplies. By simulations a concept is developed, in which effects of electromagnetic interferences are kept small and localized. The goal is to keep current loops small, to limit the switched current to the region near the switched consumer and to allow only small frequency currents to spread out further into the system. By that analogue performance can be kept high and parasitic couplings to the surrounding metal structures and other sub-detectors will be minimized. Measurements with existing prototypes support the validity of the simulations.

KEYWORDS: Voltage distributions; Large detector systems for particle and astroparticle physics; Detector grounding; Front-end electronics for detector readout

\footnotetext{
${ }^{1}$ https://twiki.cern.ch/twiki/bin/view/CALICE/WebHome

${ }^{2}$ Corresponding author.
} 


\section{Contents}

1 Motivation for power cycling for CALICE-AHCAL at the ILC 1

2 The readout ASIC as actor for power cycling 3

3 Circuits and readout boards to keep current loops localized at the actors 4

4 Charge storage at the service end $\quad 4$

5 External power supplies and the connection cables $\quad 6$

6 Performance test: operation of the SiPM-readout with power cycling 7

$\begin{array}{llr}7 & \text { Summary and outlook } & 8\end{array}$

\section{Motivation for power cycling for CALICE-AHCAL at the ILC}

The next generation of electron-positron colliders aims for precision measurements of particles generated at collision energies of $500 \mathrm{GeV}$ to $1 \mathrm{TeV}$. The acceleration of the electrons and positions to these high energies needs high gradient fields. For the international linear collider, ILC,[1] they will be generated with super conducting cavities. Although the losses at the surface of the cavities are low, it still is required, that the accelerating field is applied with a duty cycle of about $1 \%$ only. Therefore the bunches and $\mathrm{e}^{+} \mathrm{e}^{-}$-collisions will be organized in trains: For around $1 \mathrm{~ms}$ bunches follow each other every $333 \mathrm{~ns}$ and then they are absent for $199 \mathrm{~ms}$. This feature can be used in the detectors to minimize their power consumption.

The CALICE collaboration develops calorimeters to improve the energy measurements by applying the technique of particle-flow algorithm [2]. The energy of the charged particles is measured as momentum in a tracking chamber. They are summed and the energy of the neutral particles measured in the calorimeters is added. Therefore high granular calorimeters are required, which record the showers of individual impinging particles even within jets. For ILD [3] the aim is to reach a jet resolution of $30 \% / \sqrt{E_{\text {jet }}}$ to distinguish the mass difference of the $\mathrm{W}$ - and Z-bosons.

One of the CALICE developments is the analogue hadron calorimeter, AHCAL. As shown in figure 1 the calorimeter is designed as a sandwich calorimeter. A self supporting structure of stainless steel plates surrounds the beam line in an octagonal layout. Between the plates detection layers are inserted to measure the impinging particles. The sensor is composed out of small scintillator tiles of $3 \times 3 \mathrm{~cm}^{2}$, a wavelength shifting fiber and a silicon-photomultiplier, SiPM [4, 5]. Printed circuit boards, PCB, are used to support the tiles and to collect the signals from $12 \times 12$ channels to four low power ASICs [6]. This structure has to be thin, just $5.5 \mathrm{~mm}$, to keep the calorimeter compact due to the limited size of the magnet and to minimize the transversal size of the showers improving the separation of impinging particles. Such multiple PCBs are daisy chained into long 


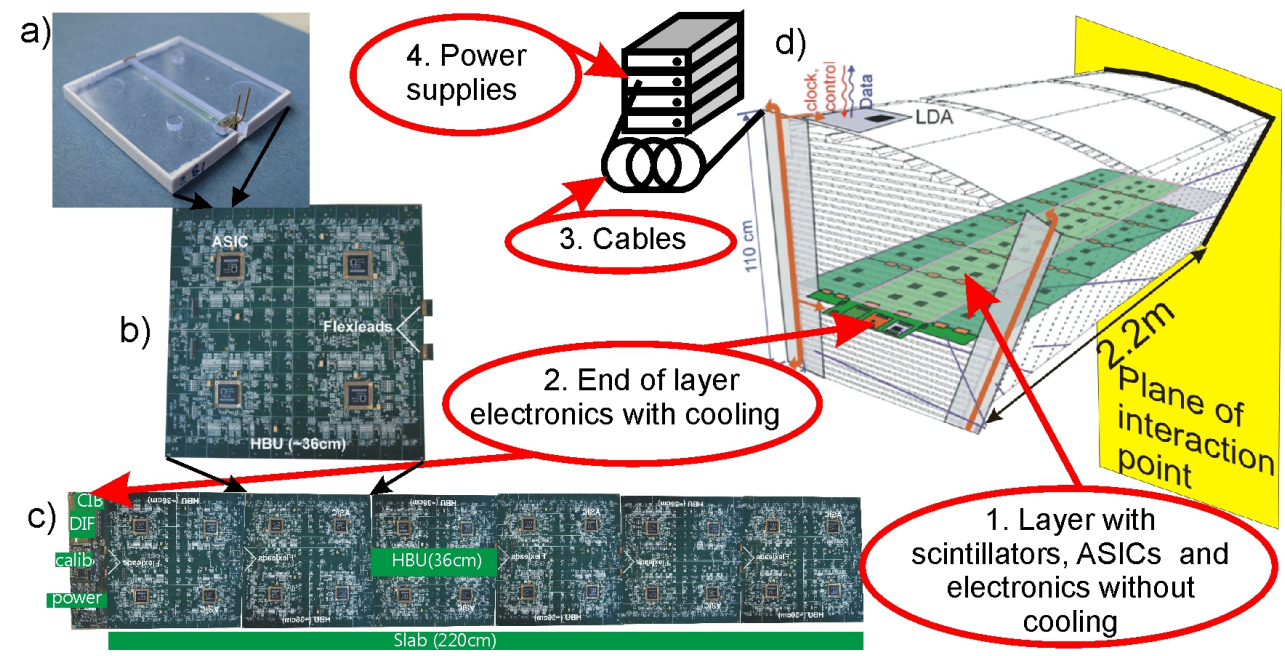

Figure 1. AHCAL from the small sensors to a full system. (a) The sensor, a $3 \times 3 \mathrm{~cm}^{2}$ scintillator with a SiPM, (b) a $36 \times 36 \mathrm{~cm}^{2}$ sized readout board for $12 \times 12$ sensors and ASICs, (c) a slab of 6 read out boards with its services at the cooled end and (d) a full octant with a stainless steel structure, 3 slabs in each layer and the items emphasized with relevance for the power cycling.

slabs and three of them are installed side by side into each detection gap. Services can be installed at the end of the octagon away from the plane through the interaction point, the service end. There additional control electronics and cooling for temperature stabilization is planned.

Parallel to the beam axis, $\mathrm{z}$, the heat is conducted within the stainless steel plates. Approximating the octagon structure as a cylinder no heat is transferred in the radial direction of the cylinder. The gaps of a sandwich calorimeter largely prevent a radial heat transfer as experienced also in other sandwich structures like HES [7] within the ZEUS calorimeter. With this simplification the temperature is described by a one dimensional time dependent differential equation:

$$
\frac{d T}{d t}=\frac{1}{\text { heat cap. } / \text { area }} \frac{P}{A}+\text { heat cond. } \frac{\partial^{2} T}{\partial z^{2}} \quad ; \quad T(t, z)=T_{\infty}\left(z^{2}-z_{0}^{2}\right)+\sum_{n=1}^{\infty} T_{n} e^{t / \tau_{n}} \cos k_{n} z
$$

The heat production of the electronics is homogeneous on the area, $\mathrm{P} / \mathrm{A}$, the end at the interaction point is a symmetry point with no heat transfer and the service end is stabilized by cooling. Therefore the temperature can be described as a static parabolic function summed with Fourier-terms in the space coordinate attenuated with an exponential time behavior. The essential parameters, the time constants, $\tau_{i}$, and the saturation temperature profiles scale with the square of the calorimeter length. Since the path for the heat transport is long, $2.2 \mathrm{~m}$, the time constants range into weeks and the temperature increase at the plane of the interaction point will only be kept at a few tenths of a Kelvin, if the power per readout channel is limited to $40 \mu \mathrm{W}$ [8]. This power budget has to be shared by the dark current of the SiPM, $15 \mu \mathrm{W}$, and the power of the readout-ASIC, $25 \mu \mathrm{W}$. Such a low power handling of fast SiPM signals, $\approx 10 \mathrm{~ns}$, can only be achieved by switching the power hungry fast analogue electronics off, when not needed. Using the train structure of the beams delivered by the accelerator for cycling the front end electronics the heat production can be reduced by a factor 100 and the homogeneity of the calorimeter need not to be compromised by cooling tubes for a liquid. 
a)

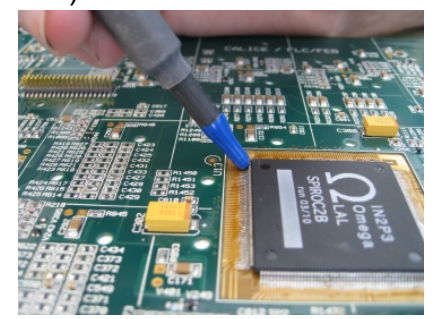

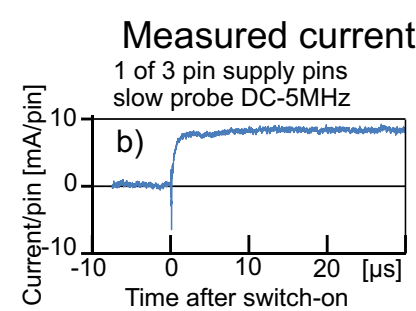

Measured currents for the preamplifier supply

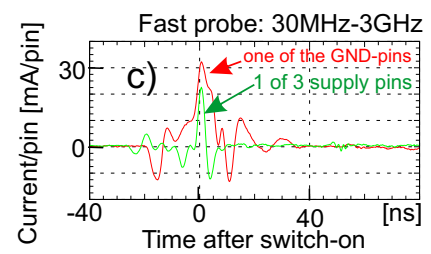

Figure 2. Measurements of the currents in the pins of the ASIC while the current sources for the supply of the preamplifiers are switched: (a) illustrates the technique of the measurements. (b) and (c) are the results of the measurements of individual pins with a slow probe also sensitive to DC-currents (b) and a high frequency bandpass coupled probe (c). Measured is always only one pin of multiple parallel used pins.

\section{The readout ASIC as actor for power cycling}

The density of the channels, one for $3 \times 3 \mathrm{~cm}^{2}$, needs a local handling of the signals and a high level of multiplexing in each layer, to minimize the connectivity to the end of the layer. For this an ASIC was developed [6] to which 36 channels are connected. Its task starts with the fine tuning of the bias voltages controlling the operation point and the gain of each SiPM individually. While the bunch train arrives the fast signals, of a few ns, from the scintillators and the SiPMs are amplified and shaped. A fast comparator generates a flag, which triggers the slower, $50 \mathrm{~ns}$ shaper, to store the signal height and time into a capacitive pipeline. This allows to store 16 signals per train adequate for the low expected occupancy at $e^{+} e^{-}$-colliders. Controlled by the electronics at the service end these stored signals are digitized with one ADC for all 36 channels of each ASIC after the end of the train. The digital signals are then multiplexed for the ASICs of each slab and transferred via the electronics at the service end to an off-detector DAQ-system during the long break between the short bunch trains.

Since not all tasks are needed for the full time, power consumption and heat production can be reduced by switching the functional blocks off when they are not needed. In particular the power hungry fast amplifiers are only needed during the bunch train, $1 \mathrm{~ms}$ or $0.5 \%$ of the time. The ADCs are only needed afterwards for $3.2 \mathrm{~ms}$. The capacitive pipeline has to be kept on during both of these tasks. The digital electronics of each ASIC is active parallel to the analogue operations and then again, when the specific ASIC is transferring its data in the schedule of the multiplexing.

The technique to realize that powering scheme is that the ASICs switch, supervised by the electronics at the service end, the current sources for the analogue parts on and off and for the digital electronics the clocks are stopped whenever possible. Hence the ASIC is the actor in the power cycling and the current consumption is changing. The switched currents are measured with magnetic field sensitive probes for individual pins (figure 2). The amplitude agrees with the expected 40mA/ASIC. Part (c) of the figure illustrates, that the generated slopes are so steep, that the frequencies are in the region of a few $100 \mathrm{MHz}$. Scaling the expectation for one ASIC to a full barrel of an AHCAL with 4 million channels the switched current is $3.4 \mathrm{kA}$.

To keep the ASICs in a stable condition the electronics on the printed circuit boards (PCBs) and the system have to stabilize the voltage and prevent switched currents from spreading out into large volumes causing disturbances within the large scale system of an ILD-detector. 


\section{Circuits and readout boards to keep current loops localized at the actors}

Within the detection layers no additional heat production is allowed. Therefore no active components can be used to stabilize the voltage and sufficient low impedance charge storage has to be provided. For the very high frequencies the charge can be stored within the layer structure of the PCB, which the ASICs and sensors are soldered to. As shown in figure 3 the supply voltage gets a nearby, $\mathrm{d}_{\text {plane }}=50-60 \mu \mathrm{m}$, ground layer providing a capacitance of $\approx 60 \mathrm{pF} / \mathrm{cm}^{2}$. The impedance of such a layer structure is simulated with a spice/VHDL-AMS based method. Each area of $1 \mathrm{~cm}^{2}$ of the $36 \times 36 \mathrm{~cm}^{2}$ large PCB is represented by a combination of a planar capacitor, resistors for the conductivity of the copper and two coils representing the inductance of a delay line in each geometrical dimension (figure 3). The capacitance of each element can be derived from the formula of a planar capacitor with the dielectric constant for the FR4 material, $\varepsilon_{r}=4$. The inductances are calculated from the travel times for a planar one dimensional wave:

$$
C_{\text {cell }}=\varepsilon_{0} \varepsilon_{r} \frac{A_{\text {cell }}}{d_{\text {plane }}}, \quad L_{\text {cell }}=\frac{1}{c_{\text {light }}^{2} \varepsilon_{0}} d_{\text {plane }}, \quad c_{\text {light }}=3 \cdot 10^{8} \frac{\mathrm{m}}{\mathrm{s}}, \quad \varepsilon_{0}=8.9 \cdot 10^{-12} \frac{\mathrm{As}}{\mathrm{Vm}}
$$

The simulated impedance of such a PCB is shown in figure 3 together with ceramic and Tantalum based capacitors described as models containing resistors and inductors for the parasitic effects. The simulation shows, that for the readout board populated with 12 Tantalum capacitors, $33 \mu \mathrm{F}$, and 17 ceramic capacitors, $1-100 \mathrm{nF}$, over a wide frequency range from $10 \mathrm{kHz}$ to $100 \mathrm{MHz}$ the ASICs get supported with an impedance below $0.1 \Omega$. In the decade above the impedance increases slowly to $0.5 \Omega$ at $1 \mathrm{GHz}$. Also the phases are away from $\pm 90^{0}$ so that the induced frequency components are dumped. For the very high frequencies the PCB structure is important, for the medium range the ceramic capacitors are needed and for the low frequencies the Tantalum. Above $1.5 \mathrm{GHz}$ the simulation can't be trusted because the discrete step of $1 \mathrm{~cm} \hat{=} 70 \mathrm{ps}$ limits the accuracy since the wavelength gets shorter than $10 \times$ the discretization step.

The simulation shows, that above $300 \mathrm{MHz}$ large jumps in the phase and impedance occurs. Increasing the resistive component between the supply and the ground gets rid of the resonances. For figure 3,16 combinations of a $1.6 \Omega$ resistors and realisticly described $100 \mathrm{nF}$ capacitors are added at different positions of the PCB and the smearing out of the resonances is demonstrated. In the experimental setup no negative effect of the resonant behavior was observed. The reason might be, that the ASICs themselves, omitted in the simulation, add the resistive behaviour. However it is easy to include the resistors in the next generation of PCBs to improve the robustness of the design.

So far the voltage supply of the ASICs is well supported above $10 \mathrm{kHz}$ and the current loops for these frequencies will be kept local on the readout boards. For the lower frequencies the impedance has to be decreased. Since that needs larger scale capacitors the space is only available at the service end. Because the wavelength for $10 \mathrm{kHz}$ is already much larger than the distance to the service end, $2.2 \mathrm{~m} \hat{=} 90 \mathrm{MHz}$, a good support from there should be possible and the currents introduced into the surrounding metal structure will be small. The next section addresses this issue.

\section{Charge storage at the service end}

The service end provides more space and cooling. This allows to install an active fast voltage regulator and capacitors (see figure 4). A setup with a FET-transistor following the regulation 
(a) Stainless steel cover on PE-GND

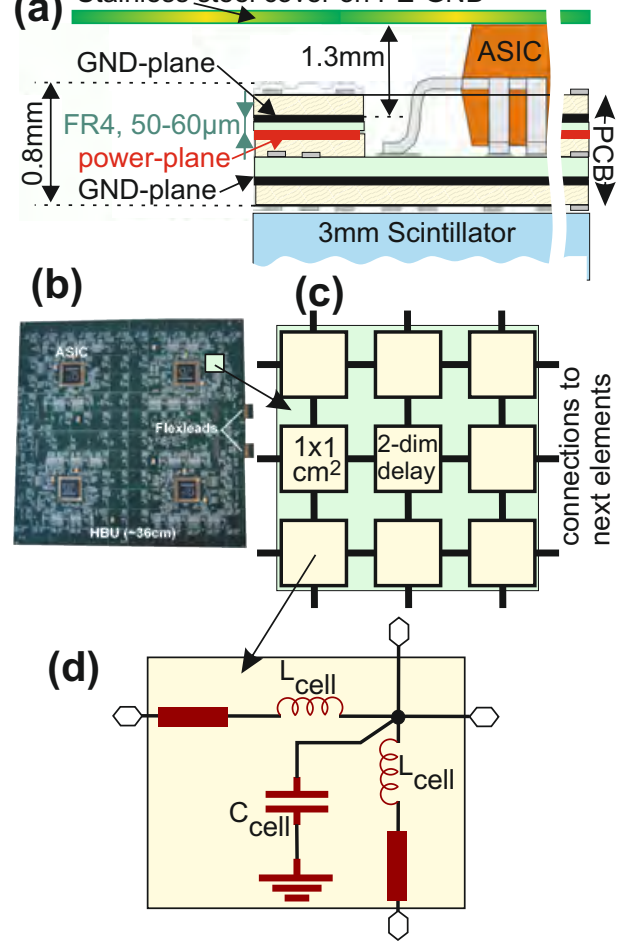

(f)

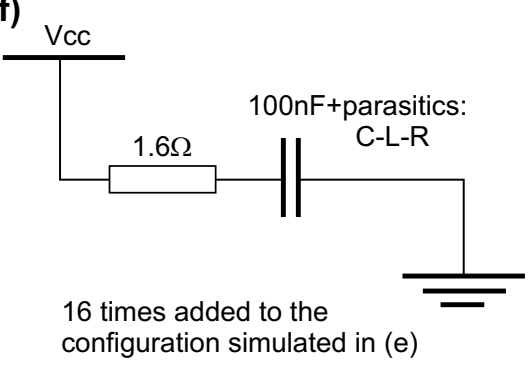

(e) Impedance of the power-GND-system for the readout board

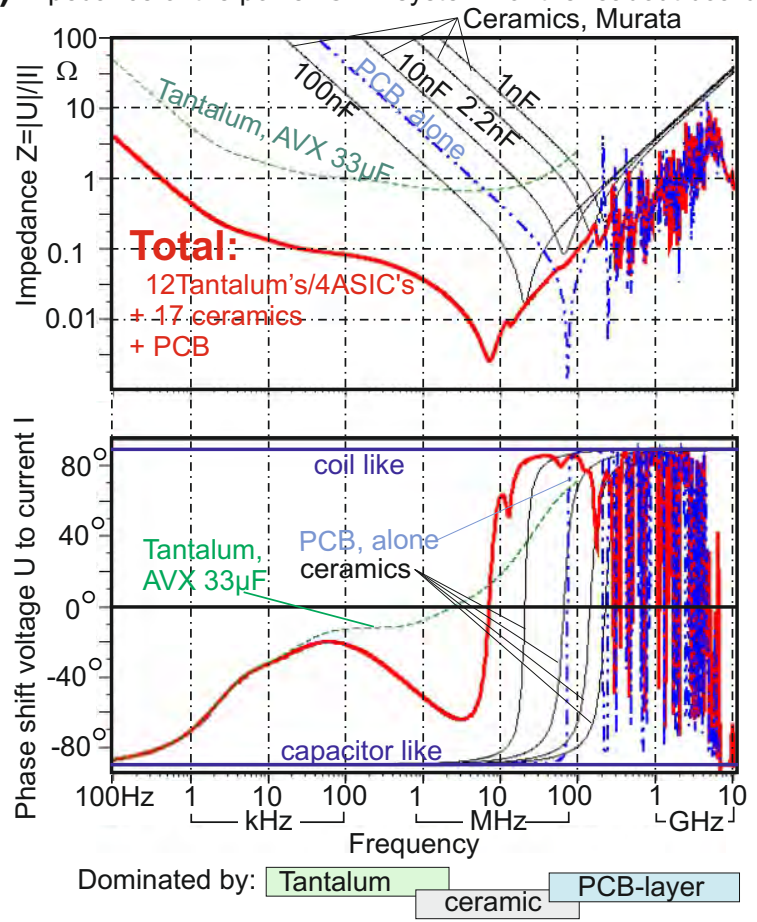

(g)

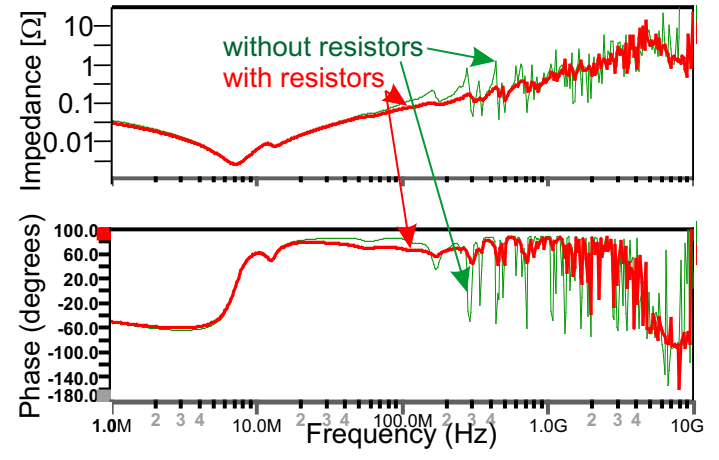

Figure 3. Impedance for providing the charge by the readout board: (a) layer structure of the PCB, (b) the PCB with the indication of a simulation element, (c) the discrete element structure for simulation, (d) the circuit for a single $1 \times 1 \mathrm{~cm}^{2}$ element appearing $36 \times 36$ per readout board, (e) the result of a simulation of the layer structure and additional capacitors, (f) and (g) adding also resistors.

circuit and a capacitor of $C_{2}=2 \mathrm{mF}$ at the output provides the fast response to requests of $2 \mathrm{~A}$ for all ASICs of one layer fully switched on during the train. The capacitor in front of the regulator $C_{3}=3.4 \mathrm{mF}$, provides the charge for the duration of the train and the digitization. Its discharge of around $0.6 \mathrm{~V}$ during a train needs a decoupling from the supply cable to avoid currents spreading into the infrastructure, and this is discussed in the next session.

The voltage at the ASIC was measured, figure 4 in a reduced setup. For the measurements only one of the readout boards was available. The measured stability of $4 \mathrm{mV}$ can be extrapolated to a full $n=6$ unit long slab. In the worst case it grows with $\frac{1}{2} n(n+1)$ to $80 \mathrm{mV}$ which would be tolerated by the ASICs. However the $2 \mathrm{~m}^{2}$ structure of each layer has a non-negligible capacitance to the surrounding ground structure of the stainless steel structure needed for the shower develop- 


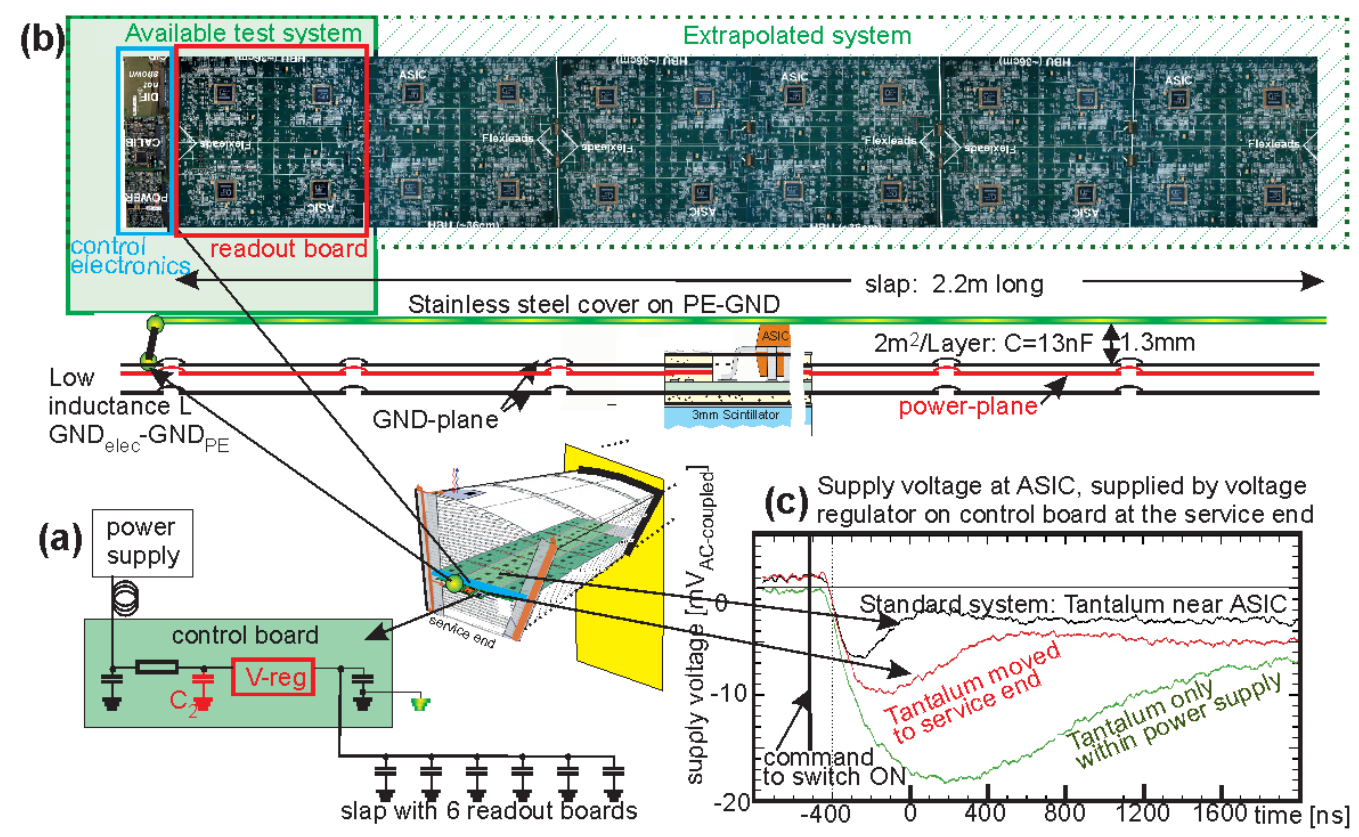

Figure 4. Concept and behaviour of the control electronics at the end of a sampling layer: (a) the concept of charge storage and voltage stabilization, (b) definition of the connection to the environmental and protection ground (PE) and (c) measurements of the stability of the voltage at the ASIC and the effect of tantalum capacitors and their distance to the ASICs.

ment. Each layer will introduce given the rise time of the voltage, $400 \mathrm{~ns}$, as measured in figure 4, a current of $<2 \mathrm{~mA}$ into the protection-ground system. That grows with the number of layers, 1500 for the AHCAL barrel, to $3 \mathrm{~A}$. But the amount of steel within the calorimeter is large and would guide the current back to the chosen grounding point at the service end. So it is not too worrying, but has to be kept in mind in continuing with the design and may need further improvements like better ground lines within the layer.

With the same setup it is also demonstrated, that the Tantalum capacitors on the readout board stabilize the voltage in a better and faster way than moving them to the service end or leaving them out. On that position thin connectors and flex foils keep the calorimeter compact. The effort to mount them on the large number of boards for readout of a full scale detector will have the advantage of less leading time after switching on, a better stabilized and more equal voltage at all ASICs and less parasitic currents within the infrastructure.

\section{External power supplies and the connection cables}

The voltage at the input of the regulation circuit was varying during the train by $0.6 \mathrm{~V}$. To understand the impact of the connection cable to an external power supply a model is setup (figure 5). The $50 \mathrm{~m}$ long cable is put $1 \mathrm{~cm}$ above a metal support as two separated wires, a not unusual way of cabling. The power supply is simulated as ideal voltage source but with a parasitic coupling of $300 \mathrm{nF}$ to its chassis as measured on a laboratory device. The cable is approximated by 20 elements of delay lines built out of L,R,C combination representing a $1 \mathrm{~mm}^{2}$ wire cross section. In 
a) supply for one layer of 18 readout units

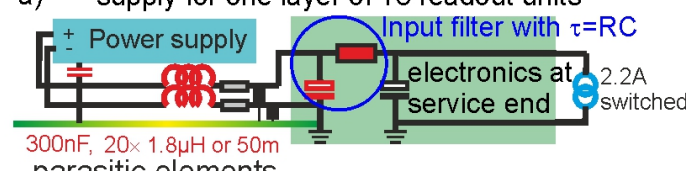

parasitic elements

c) Measured Current for 1 readout unit in supply line

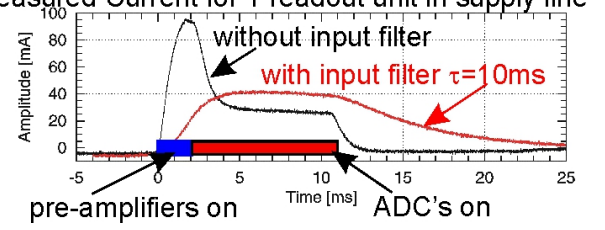

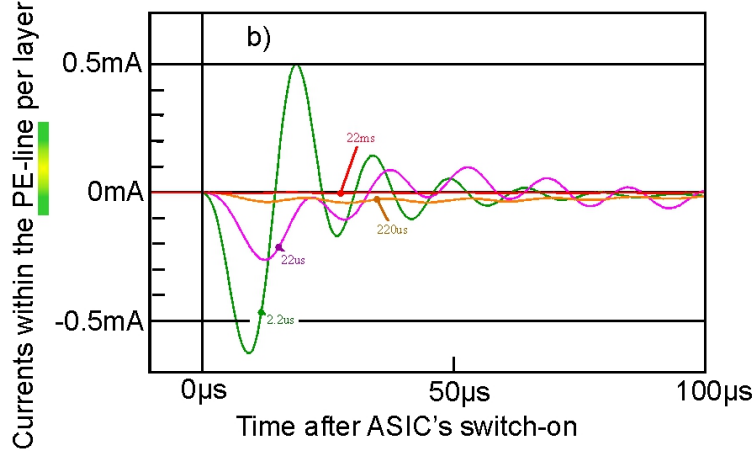

Figure 5. Influence of parasitic components of cable and power supply and the importance of an input filter: (a) the setup for simulation, (b) the simulated current in the protection ground line and (c) the measured current for a reduced setup with just one instead of 18 readout units.
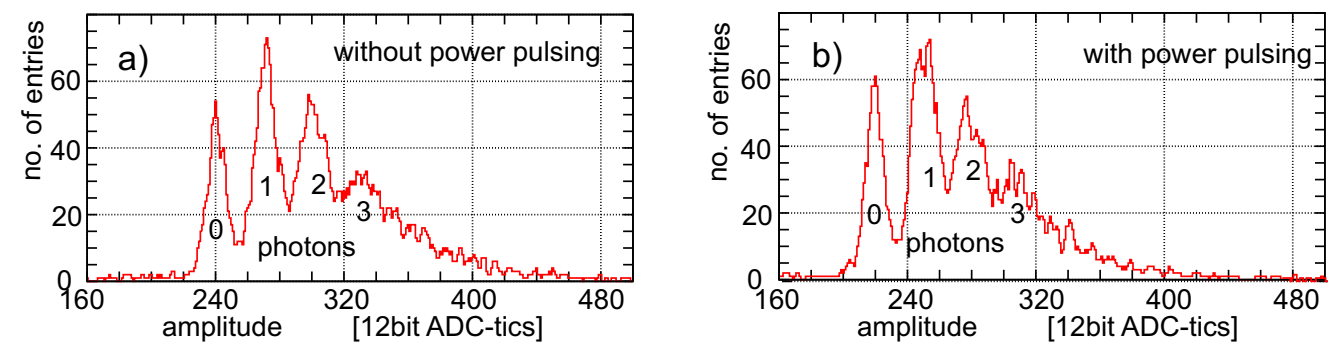

Figure 6. Single photon spectrum measured with continuous (a) and pulsed powered (b) electronics.

figure 5 simulations for different input filters at the layers are shown. The current value itself looks small, but would scale up with the number of layers to the range of one ampere and risks to disturb sensitive measurements within a full scale detector.

This is the first estimation introducing a few parasitics into the simulation. Earlier simulations without any parasitics have shown currents lower by a factor of 5000 [9]. Therefore it is advisable to look to standard EMI-effects and implement elements like $\pi$-filters into the detector, even it they take a little bit of space, and to design cabling schemes keeping supply and return close together. To this belongs also, that having chosen the grounding point near the sensitive parts at the layer end (figure 4) the external power supply should be floating, best per layer or as a compromise per group of layers.

The measurement in figure 4 demonstrates also the benefit of the filter in decreasing the peak current and rise time of the current in the supply cable itself.

\section{Performance test: operation of the SiPM-readout with power cycling}

The influence of power cycling on the noise of the readout chain was tested, by comparing the behaviour of power cycled and continuous operation. One noise sensitive parameter is the important identification of single photons with the SiPMs. Figure 6 illustrates that the noise does not change and the performance of the read out is maintained in power cycling. The baseline also shifts in other conditions like high rate operation and is under investigation by the ASIC developers. 


\section{Summary and outlook}

With simulations and measurements a strategy is worked out to understand the system setup for a power pulsed large scale experiment. Identifying current return passes and keeping the loops small compared to the wavelengths lead to low impedance supports for the current switched consumers and keep currents out of the common ground systems. Such a strategy includes all elements from the small sensors, front end PCBs, and cables to the external power supplies. Confronting future measurements with simulations will lead to an optimized system by finding the optimal compromises in effort, good EMI-behaviour and best total detector performance.

\section{Acknowledgments}

I like to thank the CALICE collaboration for the opportunity of working on this large scale project. DESY and the Helmholtz association are thanked for their support.

\section{References}

[1] G. Aarons et al., International Linear Collider, refereence design report, ILC-REPORT-2007-001 (2007).

[2] V. Morgunov, Calorimetry design with energy-flow concept (imaging detector for high-energy physics), in the proceedings of $X$ International Conference on Calorimetry in High Energy Physics (CALOR2002), March 25-29, Pasadena U.S.A. (2002).

[3] H. Stoeck et al., International Large Detector - Letter of intent, arXiv:1006. 3396 (2010).

[4] G. Bondarenko et al., Limited Geiger-mode silicon photodiode with very high gain, Nucl. Phys. B 61 (1998) 347.

[5] B. Dolgoshein et al., Status report on silicon photomultiplier development and its applications, nucl Instrum Meth. A 563 (2006) 368.

[6] L. Raux et al., SPIROC measurement: Silicon Photomultiplier Integrated ReadOut Chip for ILC, IEEE Nucl. Sci. Symp. 2008 (2008).

[7] P. Göttlicher, The ZEUS Hadron Electron Separator, Performance and Experience, in the proceedings of the X International Conference on Calorimetry in High Energy Physics (CALOR2002), March 25-29, Pasadena U.S.A. (2002).

[8] P. Göttlicher, System aspects of the ILC-electronics and power pulsing, in the proceedings of Topical Workshop on Electronics for Particle Physics (TWEPP07), September 3-7, Prague, Czech Republic (2007).

[9] P. Göttlicher, A concept for power cycling the electronics of CALICE-AHCAL with the train structure of ILC, Phys. Proc. 37 (2012) 1586. 